\title{
Behavioral and biological risks of women seeking HIV test in an anonymous testing center
}

\section{Authors}

Ayrton Daniel Ribeiro Filho ${ }^{1}$ Paulo César Giraldo ${ }^{2}$

Maria José Penna

Maisonnette de Attayde

Silva ${ }^{3}$

Rose Luce Gomes do Amaral $^{4}$

José Eleutério Junior ${ }^{5}$ Ana Katherine da Silveira Gonçalves ${ }^{6}$

${ }^{1} \mathrm{MD}, \mathrm{PhD}$, Universidade Estadual de Campinas

(Unicamp), Brazil

${ }^{2}$ Post-doctorate, Weill Cornell Medical College,

Cornell University, Division

of Microbiolgy and

Immunology; Head Professor,

Tocogynecology Department,

Unicamp, Brazil

${ }^{3} \mathrm{MSc}$ in Health Sciences,

Universidade Federal

do Rio Grande do Norte

(UFRN); Assistant Professor,

Universidade Potiguar, Brazil

${ }^{4} \mathrm{MD}, \mathrm{PhD}$ in Gynecology,

Unicamp; Physician,

Tocogynecology Department,

Unicamp, Brazil

${ }^{5} \mathrm{PhD}$ in Gynecology, Unicamp; Professor, Tocogynecology Department, Universidade Federal do Ceará, Brazi ${ }^{6} \mathrm{PhD}$, Unicamp; Professor, Tocogynecology Department, UFRN, Brazil

Submitted on: 01/04/2011 Approved on: 02/17/2011

Correspondence to: Paulo C. Giraldo

Universidade Estadual de Campinas

Cidade Universitária

"Zeferino Vaz"

Rua Alexander Fleming, 101, Campinas

13083-881, São Paulo, Brazil

Phone/Fax: (19) 3788-9306. giraldo@unicamp.br

We declare no conflict of interest.

(ㅇ)2011 Elsevier Editora Ltda. All rights reserved.

\begin{abstract}
Due to the high prevalence and morbidity sexually transmitted diseases are highly relevant to public health, especially for women. Objectives: To determine and compare the behavioral and biological risks associated with human immunodeficiency virus acquisition. Methods: A group of 253 women who voluntarily sought anonymous testing were interviewed to find out their behavioral risk. Biological risk was identified by means of gynecological exam, colposcopy as well as blood and cervicovaginal sampling for serological and microbiological exams. Using known traditional risk factors, a table of scores classified the subjects into high, low and absent for behavioral and biological risks. Frequency and percentage of each risk was tabulated and the correlation between risks was obtained by calculating the Kappa statistic. Results: $79.8 \%$ of subjects were found to have behavioral risks, and $79.1 \%$ biological risks. It was also found that $66.7 \%$ of the women (169) with high behavioral risk also had high biological vulnerability. However, 31 out of 51 women without any behavioral risk had biological vulnerability $12.2 \%$. The Kappa statistic demonstrated low agreement between the latter risks $[\mathrm{K}=0.0595 \% \mathrm{CI}(-0.06$ to 0.17$)]$. Conclusion: Women who seek care in centers for anonymous testing have high biological risk, which is neither proportional nor concurrent to behavioral risk. The low concordance found between these risks suggests the need for routine gynecological investigation (clinical and microbiological) for all women.
\end{abstract}

Keywords: HIV; sexually transmitted diseases; women.

\section{INTRODUCTION}

The acquired immune deficiency syndrome (AIDS) was identified almost 20 years ago and quickly became one of the leading causes of death worldwide. The World Health Organization indicates that the human immunodeficiency virus (HIV) has infected nearly 33.2 million people. An annual incidence of 2.5 million and a mortality of 2.1 million are estimated. ${ }^{1,2}$ An evaluation of the epidemiological history of HIV infection reveals that initial infection spread rapidly among the sexually exposed, suggesting an association between HIV and behavioral factors. ${ }^{3}$

Several behavioral factors have been recognized and accepted as HIV infection facilitators: ${ }^{4-7}$ a history of multiple sexual partners, sexually transmitted diseases (STDs), unstable marital status, use of intravenous drugs, sex with HIV-positive partners, first sexual experience prior to 14 years of age, use of oil based lubricants, practice of anal sex, being under 45 years of age, inconsistent use of condoms, recent use of intrauterine device, sex under the influence of alcohol, as well as sex involving traumatic bleeding or during menstruation..$^{3-7}$

Although high-risk sexual behavior seems to be directly associated with the increase of STD/AIDS, ${ }^{7}$ it is not the only cause for the spread of AIDS, as women with low-risk behavior are also infected by HIV. ${ }^{6}$ It is important to note that sexual behavior is not the only risk factor for HIV acquisition. Vulvovaginitis, cervicitis, HPV, genital ulcers, and use of illicit drugs also play a role in HIV transmission. ${ }^{8-12}$

Clinical conditions like vulvovaginal ulcers, excoriations, cracks, cervicitis, or coinfections (Chlamydia trachomatis, herpes simplex, human papilloma virus - HPV) can all increase the risk of STD/AIDS. ${ }^{8-11}$ Inflammatory nonulcerative genital conditions such as vulvovaginitis, cervicitis and pelvic inflammatory disease have also been shown to be important 
risk factors. Another consideration is that diagnosis of a sexually transmitted infection should increase suspicion of other STDs. ${ }^{8-11}$

The prevalence of HIV infection is increasing among women of all ages and regions, thus reducing the male/female ratio to $3 / 2 .{ }^{10}$ Several epidemiological studies show a growing number of HIV infected women. In recent years heterosexual transmission has been identified as the most important means of infection ${ }^{7}$ and women with the fastest growing incidence. This could be due to untreated genital infections: adequate treatment of such infections would provide the opportunity to restore the vaginal flora as well as the integrity of the genital mucosa, thus reducing the risk of HIV acquisition. ${ }^{89,11}$

In order to reduce the risk of female HIV infection, several studies have established the necessity of an active search for other parallel diseases, in addition to serological testing and behavioral counseling. ${ }^{1,2,12,13}$ In Africa, Laga et al. ${ }^{14}$ reported that the treatment of genital disorders could reduce the rate of HIV infection in HIV-exposed people. The purpose of this study was to determine if there is any correlation between biological and behavioral risks for acquisition of HIV infection in women. If correlation between biological and behavioral risks is poor, as expected, the knowledge could help create new strategies to decrease the transmission and acquisition of HIV/AIDS.

\section{MATERIAL AND METHODS}

A cross-sectional study involving 253 women analyzed the association between behavioral and biological risks associated with HIV infection. Women who spontaneously came to the Center of Anonymous Testing (CAT) located in downtown Campinas, São Paulo State, Brazil, a city of approximately one million inhabitants, were invited to take part in the study. CATs are outpatient clinics distributed throughout Brazil. They consist of medical clinics without a lab or any other structure for treatment or diagnosis, where blood samples are taken and gynecological exams are freely provided to all patients. Patients who attend these clinics feel threatened by the possibility of hosting HIV or any kind of STD.

Women included in the study underwent a gynecological evaluation and had blood drawn for serological and microbiological tests. Women who were on systemic or topical antibiotics, as well as new or expecting mothers were excluded.

After signing the term of free informed consent, all patients were interviewed and examined by the principal investigator in a gynecology office at the CAT. The interview lasted for an average of one hour and included 20 questions on topics such as demographic information (age, educational and economic level) and sexual behavior (oral sex, anal sex, condom use, number of partners, STDs, drug use, etc.).
Eight milliliters of blood were drawn from each patient forearm, centrifuged and the plasma sent to the Laboratory of Immunology and AIDS and checked for syphilis (VDRL and ELISA), HIV, hepatitis B, C and E.

Using the ELISA technique, the serological tests for hepatitis $\mathrm{B}, \mathrm{C}$ and $\mathrm{E}$ (anti-HBc, anti-HCV, anti-HEV and $\mathrm{HBs} \mathrm{Ag}$ ) were performed in the hepatitis study group laboratory/Unicamp. Serological tests: anti-HAV IgM and anti-HBc and HBsAg IgM were detected by EIA (Abbott Laboratories, Abbott Park, IL, USA, and Wiesbaden-Delkenheim, Germany). Anti-HCV was tested using a secondgeneration EIA (Abbott Laboratories). Anti-HEV IgM and anti-HEV IgG were assayed by EIA (Genelabs Diagnostics, Singapore, and Abbott Laboratories, respectively). All assays were performed in duplicate according to the manufacturer instructions.

Genital infections caused by CT, NG, or HPV were diagnosed by sampling the uterine cervix by second-generation Digene ${ }^{\circledast}$ Hybrid Capture Assay in preserved cervical-vaginal material in Universal Collection Medium (UCM, Digene ${ }^{\circledast}$ ) as recommended by the manufacturer. Positive results were retested for identification of CT-DNA, NG-DNA and HPVDNA in the specimen. Positive and negative controls were performed in triplicate. All tests were duplicated, and cases reaching the values specified by the manufacturer were considered positive.

Vaginal secretion was collected from the upper third side using a sterile spatula and Gram stained to look for the presence of bacterial vaginosis. Diagnosis of abnormal vaginal flora was defined by the Nugent criteria, ${ }^{15}$ while candidiasis was diagnosed by the presence of vaginal hyphae and trichomoniasis by the presence of flagellate.

In this study, the presence of behavioral risk was assessed according to the literature: present age, obstetrical history, contraceptive method, age at first sexual intercourse, number of sexual partners over the past five years, high-risk sexual partners, history of fellatio or anal sex, irregularity of condom use, sexual practice with an HIV positive partner, drug injection user, history of STDs, prior consultation at CAT, as well as previous surgery and gynecological complaints.

The presence of biological risk took in consideration the following factors, as previously defined: presence of cervicitis (Chlamydia and gonococcus) and vulvovaginitis (candidiasis, trichomoniasis), abnormal vaginal flora (bacterial vaginosis), vaginal ulcers, HPV infection, all findings from clinical and gynecological exams, as well as laboratory tests. ${ }^{8-11}$

Concordance of biological and behavioral risks was tested by the weighted kappa statistic. ${ }^{16,17}$

This study was approved by the institutional ethics committee and followed the Guiding Medical Doctor recommendations of the Biomedical Research Involving Human Subjects and the Declaration of Helsinki. ${ }^{18}$ 


\section{RESULTS}

The mean age of subjects was 29.1 years $( \pm 9.1)$; demographic data of study subjects are found in Table 1. Sexual characteristics related to behavioral risks are listed in Table 2. Age at first sexual intercourse was shown to be 17.2 years ( \pm 3.4 ), while contraception was used by $91.8 \%$, and $35.1 \%$ of the cases were nulliparous. In the sample $35.9 \%$ had had between one and two pregnancies, $14.6 \%$ had a history of STD, 7.9\% had an HIV-infected partner and $1.6 \%$ of the women admitted to be drug addicts. It was observed that $79.8 \%$ of these women had at least one behavioral risk for HIV infection. Approximately 30\% of patients had been sexually active with more than five partners in the past five years, and $44 \%$ were not monogamous. In addition, all women used condoms irregularly, $88 \%$ with a fixed partner and $58.4 \%$ with different partners. The practice of anal sex was reported by $32.8 \%$ women, and $14.6 \%$ reported a history of STD while $19.0 \%$ had had a partner at risk.

Table 2 shows that there was one or more biological risks (biological vulnerability) found in 79.1\% (200/253) of the women studied. The most common were abnormal vaginal microflora (bacterial vaginosis) (43.1\%), cervicitis (Chlamydia and gonococcus) (17.0\%), vulvovaginitis (candidiasis and trichomoniasis) (17.0\%) and vaginal ulcers (8.3\%).

Only five women were found to have HIV infection: four of them had behavioral risk (Table 1) while three had biological risk (Table 2). Two subjects presented both types of risks, while one only behavioral, and two only biological.

Table 3 shows that both an elevated frequency of behavioral risk, 79.8\% (202/253), and biological risk, 79.1\% (200/253), were found among the women studied. It was also noticed that $66.7 \%$ of the women (169) who had high behavioral risk also had high biological vulnerability; however, 31 out of 51 women without any behavioral risk had biological vulnerability. Agreement between behavioral and biological risks assessed the weighted kappa coefficient used the categories proposed by Landis \& Koch. ${ }^{19}$ Updated support for the choice of the kappa coefficient was provided by Viera et al. ${ }^{17}$ In this study the correlation between the behavioral risk and biological risk was found to be low or negligible [weighted kappa coefficient $=0.05$ $95 \%$ CI $(-0.06$ to 0.17$)]$.

\section{DISCUSSION}

Evaluating the epidemiological history of HIV infection, it appears that initially there was a rapid spread of infection in the population of greater sexual exposure, explained by the causal relationship between HIV and behavioral factors. ${ }^{3-7}$ There is no doubt that sexual risk is directly associated with increased STDs. However, this alone could not be responsible for the spread of STDs, especially in populations with low risk behavior. It is of utmost importance to recognize
Table 1. Behavioral risks for HIV infection among 253 women attending an anonymous test center for AIDS

\begin{tabular}{|lcc|}
\hline $\begin{array}{l}\text { Total behavioral risk } \\
\text { Irregular use of condom } \\
\quad \text { (monogomous) }\end{array}$ & 170 & $88.0^{\mathrm{a}}$ \\
\hline $\begin{array}{l}\text { Irregular use of condom } \\
\quad \text { (non-monogomous) }\end{array}$ & 66 & $58.4^{\mathrm{b}}$ \\
\hline Fellatio & 14 & 56.9 \\
\hline Infrequently non-monogamous & 113 & 44.4 \\
\hline Frequently non-monogamous & 60 & 23.7 \\
\hline Sexual partners $\geq 5$ (five years) & 85 & 33.1 \\
\hline Anal sex & 83 & 32,8 \\
\hline Previous history of STD & 37 & 14.6 \\
\hline Sexual partner with high risk for HIV & 48 & 19.0 \\
\hline HIV + partner & 20 & 7.9 \\
\hline Use of injected drugs & 04 & 1.6 \\
\hline
\end{tabular}

${ }^{a} 170$ cases of 193 monogomous women (only one sexual partner).

${ }^{\mathrm{b}} 66$ cases of 113 non-monogamous (several sexual partners).

Table 2. Biological risk for HIV infection among 253 women attending an anonymous test center for AIDS

\begin{tabular}{lcc}
\hline Total biological risk & $\mathbf{2 0 0}(\mathbf{n})$ & $\mathbf{7 9 . 1}(\mathbf{\%})$ \\
\hline $\begin{array}{l}\text { Abnormal vaginal microflora } \\
\text { (bacterial vaginosis) }\end{array}$ & 109 & 43.1 \\
\hline Cervicitis (Chlamydia and gonococcus) & 43 & 17.0 \\
\hline Vulvovaginitis (candidisis) & 43 & 17.0 \\
\hline HPV infection & 22 & 8.7 \\
\hline Vaginal ulcers & 21 & 8.3 \\
\hline Asymptomatic & 151 & 59.3 \\
\hline
\end{tabular}

Table 3. Distribution of biological and behavioral risk among women attending an anonymous test center for AIDS

\begin{tabular}{lcc}
\hline \multirow{2}{*}{$\begin{array}{l}\text { Total behavioral } \\
\text { risk }\end{array}$} & \multicolumn{2}{c}{ Biological risk } \\
& $\begin{array}{c}\text { Positive } \\
\text { (79.1\%) }\end{array}$ & $\begin{array}{c}\text { Negative } \\
53(20.9 \%)\end{array}$ \\
\hline Positive 202(79.8\%) & $169(66.7 \%)$ & $33(13 \%)$ \\
\hline Negative $51(20.1 \%)$ & $31(12.2 \%)$ & $20(7.9 \%)$ \\
\hline
\end{tabular}

*Kappa coefficient $=0.05$ (-0.06 to 0.17$)$.

$*$ *Kappa coefficient of $\leq 0.20=$ minimal correlation between behavioral and biological risk. 
the social and biological mechanisms involved in increasing STDs vulnerability in low-risk populations, since the majority of infected women in Brazil present low-risk behavior.

In the 1990s, it was believed that data gathered from anamnesis would create the possibility to define female behavioral groups most likely to be exposed to HIV infection. However, it is not clear whether this behavioral risk represents a biological risk as well. Clarification on this point could help developing protocols, helping doctors and the general population to identify the risks.

It was observed that only $66.7 \%$ of women with behavioral risk were also biologically vulnerable, $13 \%$ presented behavioral risk without biological risk and $12.2 \%$ suffered biological risk without behavioral risk. It was observed in this study that the correlation between behavioral risk and biological vulnerability scarcely existed. As a result, HIV infection is a possibility not only for women with high behavioral risk, but also for those with low behavioral risk and high biological risk. This conclusion is also supported by the fact that only five women were found to be infected, however discordant: those presenting behavioral risks did not necessarily present biological risks, and vice versa. This being said, no specific group is targeted within the general population, concluding that all women are vulnerable. It is important for national health programs to determine the overlap between the two types of risk in order to help health officials determine which groups should receive interventions to prevent HIV infection.

This lack of correlation between behavioral and biological vulnerability causes difficulties when identifying the profile of women who would benefit most from gynecological appointments. Thus, a routine check-up is also important for asymptomatic and low risk women.

Considering the observed aspects, gynecological consultation with genital microbiological evaluation seems to be essential for asymptomatic women with low-risk behavior, since we observed a high prevalence of biological vulnerability in patients with low-risk behavior. In this study it was found that $59.3 \%$ of women who had biological vulnerability were asymptomatic: confirming the absence of behavioral risk does not rule out risk of HIV infection due to biological factors.

Biological vulnerability has already been described in some groups of women. Studying women in a rural community of South Africa, it was observed that $61 \%$ of asymptomatic women attending a family planning clinic suffered from genital alterations. ${ }^{19}$ Similar results were found in $25 \%$ of women seen in a family planning clinic in Texas (USA). ${ }^{20}$ In Brazil an epidemiological study of genital alterations commonly seen in primary health care was done after having observed a high prevalence of genital lesions in asymptomatic women. ${ }^{21}$

Asymptomatic women with high biological risk and low behavioral risk seem to be common. However, the present report is the first in which the women felt at risk, and as a result sought an anonymous HIV testing center. Considering this, we can say that our results are unique.
The greatest difficulty for HIV health programs aiming to decrease the rate of infection is reducing poverty, thus increasing prevention campaign results..$^{22}$ Lopman et al. ${ }^{23}$ in a Zimbabwe study found that HIV incidence and mortality rates, and perhaps behavioral risk, were higher in lower socioeconomic groups. The latter, similar to the present study, also found no relationship between behavioral factors and higher STD biological vulnerability. Another study of HIVinfected Brazilian women supported these findings by concluding that the majority of subjects $(55.6 \%)$ had a single sexual partner during the five-year period. ${ }^{24}$

The results of our study were somewhat unexpected. The literature shows that a history of STDs is a marker for other STDs. ${ }^{8,911}$ Thus, it was expected that these women would present high prevalence of facilitating factors for STDs. Nonetheless, in this investigation having had a history of STDs reduced biological vulnerability. The most plausible explanation for this finding is that some women who had suffered a psychological trauma as a consequence of the STD diagnosis and treatment seem to have adopted safer behavioral habits afterwards, and thus may have incorporated into their lives the importance of a preventive gynecological routine. This supposition reinforces the hypothesis that information on prevention through gynecological control can assist in the creation of substantial behavioral modifications allowing for safer sexual practices.

The greatest limitation of this study is the fact that it is cross-sectional: the investigator had contact with the subjects only once, thus removing the possibility of establishing a trusting relationship with the patients, reducing the potential for greater confidence and veracity on the part of the patients. Another limiting aspect is inherent to the subjective approach of the questionnaire and the clinical assessment, providing low reproducibility. Finally, the structure of the CAT outpatient clinics limited this study to certain tests, some not being the most appropriate for the desired results.

Despite these limitations, it is possible to consider that women with high behavioral risk are not necessarily at higher risk of infection than those with high biological risk. Even though disassociated, both types of risk can either be encountered in one individual or not. In conclusion, no variable seems capable to identify the risk for HIV infection.

\section{REFERENCES}

1. Webber G. The impact of migration on HIV prevention for women: constructing a conceptual framework. Health Care Women Int 2007; 28:712-30.

2. Swamy M. UN agencies issue new guidelines for HIV testing. HIV AIDS Policy Law Rev 2007; 12:39-40.

3. Muruka C, Mruka A. Guidelines for environmental health management in children's homes in Sub-Sahara Africa. Int J. Environ Res Public Health 2007; 4:319-31.

4. Beyrer C. HIV epidemiology update and transmission factors: risks and risk contexts - 16th International AIDS Conference epidemiology plenary. Clin Infect Dis 2007; 44:981-7. 
5. Patinkin N, Werner B, Yust I, Yagil Y, Drory M, Burke M. An investigation of the practice of unsafe sex yet repeated HIV testing. Soc Work Health Care 2007; 44:73-90.

6. Bozicevic I, Stulhofer A, Adjukovic D, Kufrin K. Patterns of sexual behavior and reported symptoms of STI/RTIs among young people in Croatia-implications for interventions' planning. Coll Antropol 2006; 30:63-70.

7. Leichliter JS, Chandra A, Liddon N, Fenton KA, Aral SO. Prevalence and correlates of heterosexual anal and oral sex in adolescents and adults in the United States. J Pediatr Adolesc Gynecol 2007; 20:225-31.

8. McClelland RS, Richardson BA, Hassan WM et al. Improvement of vaginal health for Kenyan women at risk for acquisition of human immunodeficiency virus type 1 : results of a randomized trial. J Infect Dis 2008; 197:1361-8.

9. Averbach SH, Gravitt PE, Nowak RG et al. The association between cervical human papillomavirus infection and HIV acquisition among women in Zimbabwe. AIDS 2010; 24:1035-42.

10. Sutcliffe CG, Aramrattana A, Sherman SG et al. Incidence of HIV and sexually transmitted infections and risk factors for acquisition among young methamphetamine users in northern Thailand. Sex Transm Dis 2009; 36:284-9.

11. NIMH Collaborative HIV/STD Prevention Trial Group. Sexually transmitted disease and HIV prevalence and risk factors in concentrated and generalized HIV epidemic settings. AIDS 2007; 21:81-90.

12. Michelo C, Sandøy IF, Dzekedzeke K, Siziya S, Fylkesnes K. HIV prevalence declines among young people in select Zambian communities: population-based observations (1995-2003). BMC Public Health 2006; 10:279.

13. Workowski KA, Berman SM. Center for Disease Control e Prevention, Sexually transmitted diseases treatment guideline. MMWR Recomm Rep 2006; 55:1-94.

14. Laga M. STD control for HIV prevention- it works! Lancet 1995; 346:518-9.
15. Schwebke JR, Hillier SL, Sobel JD, McGregor JA, Sweet RL. Validity of the gram stain for the diagnosis of bacterial vaginosis Obstet Gynecol 1996; 88(4):573-6.

16. Landis JR, Koch GG. The measurement of observer agreement for categorical data. Biometrics 1977; 33:159-74.

17. Viera AJ, Garrett JM. Understanding interobserver agreement: the kappa statistic. Fam Med 2005; 37:360-3.

18. Declaración de Helsinki. Bol Sanit Panam 1990; 108:626-37.

19. Wilkinson D, Ndovela N, Harrison A, Lurie M, Connolly C, Sturm AW. Family planning services in developing countries: an opportunity to treat asymptomatic and unrecognized genital tract infections? Genitourin Med 1997; 73:558-60.

20. Poindexter AN, Frank ML, Villarreal G, Johnson ML. Risk factors for infection with human immunodeficiency virus among low-income women undergoing voluntary sterilization. Tex Med 1992; 88:70-4.

21. Giraldo PC, Moreira Filho DC, Nunes ECP, Guimarães RCA. Contribuição a estudo epidemiológico das ginecopatias prevalentes no atendimento primário de saúde. Rev Inst Mat Inf 1987; 1:155-60.

22. De Walque D, Nakiyingi-Miiro JS, Busingye J, Whitworth JA. Changing association between schooling levels and HIV-1 infection over 11 years in a rural population cohort in southwest Uganda.Trop Med Int Health 2005; 10:993-1001.

23. Lopman B, Lewis J, Nyamukapa C, Mushati P, Chandiwana S, Gregson S. HIV incidence and poverty in Manicaland, Zimbabwe: is HIV becoming a disease of the poor? AIDS 2007; 21:57-66.

24. Amaral E, Faúndes A, Gonçales NS, Pellegrino Júnior J, de Souza CA, Pinto e Silva JL. Prevalence of HIV and Treponema pallidum infections in pregnant women in Campinas and their association with socio-demographic factors. Sao Paulo Med J 1996; 114:1108-16. 